$\xi=$ 줄

\title{
Gingival crevicular fluid level of soluble triggering receptor expressed on myeloid cells-1(strem-1) in periodontal health and disease: a case-control study
}

\author{
SurabhiGigras $^{1 *}$, Sudhir R Patil ${ }^{2}$, Veena HR $^{3}$, SnehaDani ${ }^{4}$ \\ ${ }^{1}$ Post-graduate student, Department of periodontics, KLE Society's Institute of Dental Sciences, Bengaluru, India \\ ${ }^{2}$ Head of department, Department of periodontics, KLE Society's Institute of Dental Sciences, Bengaluru, Karnataka, India \\ ${ }^{3}$ Reader, Department of periodontics, KLE Society's Institute of Dental Sciences, Bengaluru, Karnataka, India \\ ${ }^{4}$ Senior Lecturer, Department of periodontics, KLE Society's Institute of Dental Sciences, Bengaluru, Karnataka, India \\ *Corresponding author E-mail: gigrassurabhi@gmail.com
}

\begin{abstract}
Background: The Triggering Receptor Expressed On Myeloid Cells-1(TREM-1) is a cell-surface receptor of the immunoglobulin superfamily and found to be involved in the amplification of the inflammatory response to various microbial infections, including periodontal diseases.

Objectives: The present study was designed to examine gingivalcrevicular fluid(GCF) levels of soluble TREM-1 (sTREM-1) levels in periodontal health and disease as well as evaluate the effect of scaling and/or root planing on the same.

Methods: Based on gingival index, probing pocket depth, clinical attachment level, and radiologic parameters (bone loss), 45 subjects were initially divided into three groups- Group, I (Periodontally healthy), Group II (Gingivitis) and Group III (Chronic Periodontitis).From each of the subjects, GCF sample was collected at baseline and scaling and/or root planing was instituted in group II and group III patients. GCF samples were subsequently collected at eight-week interval. Levels of sTREM-1 in collected GCF samples were estimated using enzyme-linked immunosorbent assay.

Results: The lowest GCF levels of sTREM-1 were found in periodontal health $(69.50 \pm 1.8 \mathrm{pg} / \mathrm{ml})$ followed by gingivitis $(257.17 \pm 79 \mathrm{pg} / \mathrm{ml})$ and chronic periodontitis $(3658.14 \pm 55 \mathrm{pg} / \mathrm{ml})$ in increasing order, suggesting that levels of sTREM-1 in crevicular fluid increased with the severity of periodontal disease. sTREM-1 levels decreased significantly from baseline to the end of 8 weeks following non-surgical periodontal therapy.

Conclusion: Increased GCF levels of sTREM-1 from periodontal health to disease strengthen its association with periodontal status.
\end{abstract}

Keywords: Chronic Periodontitis; Gingival Crevicularfluid; Gingivitis; Scaling and/or Root Planing; Triggering Receptor Expressed on Myeloid Cells-1

\section{Introduction}

Periodontal diseases are one of the most common polymicrobialinfections in humans, characterized by persistent inflammation, connective tissue breakdown and alveolar bone destruction. Subjective symptoms are typically mild during the early phase of disease, and subjects tend to ignore the condition until more severe symptoms appear. Thus, early diagnosis of periodontal diseases is important with respect to quality of life later.

Traditional diagnostic procedures (clinical as well as radiographic) can assess only disease history; current diseases status can not be analysed. So, there is a need through the development of new diagnostic tests that can detect the presence of active disease, predict future disease progression and evaluate the response to periodontal therapy(Anitha K et al 2013 p.59). Since GCF is an inflammatory exudate that reflects ongoing events in the periodontal tissues that produce it, an extensive search has been made for its components that might serve as potential diagnostic or prognostic markers for the progression of periodontitis. Recently, another class of cell-surface receptors, "Triggering Receptor Expressed On Myeloid cells-1 (TREM-1)" has been identified in gingival crevicular fluid (GCF) and associated with periodontal disease pathogenesis (Bostanci et al 2011 p.387; Bison C et al 2012 p.1141; Bostanci N et al 2013 p.37; Lei L et al 2013 p.88; Willi M et al 2014 p.190).

TREM-1 is a cell-surface receptor of the immunoglobulin superfamily, which is constitutively expressed on the surface of human polymorphonuclear neutrophils (PMNs) and monocytes. It gets activated upon bacterial recognition by host cells via toll like receptors and triggers a number of intracellular signalling events that result in enhanced pro-inflammatory cytokine production. Bacterial or fungal infections can cause up-regulation of membrane-bound TREM-1 as well as release its soluble form (sTREM1), rendering it a useful early inflammatory biomarker for systemic infections (Bostanci N et al 2013 p.1). Since TREM-1 amplifies the inflammatory response induced by pathogenic bacteria, it may serve as a useful marker for detecting the progression of periodontal lesions.

The study of GCF levels of sTREM-1 may provide useful insight into the pathogenesis and may improve diagnostic and therapeutic approaches in the treatment of patients with advanced periodontitis. This study is, therefore, an attempt to estimate and compare GCF levels of sTREM-1 in periodontally healthy, gingivitis and chronic periodontitis subjects and evaluate the effect of non- 
surgical periodontal therapy in the form of scaling and/or root planing on the same.

\section{Methods}

\subsection{Study population}

The study was performed on 45 subjects diagnosed as periodontallyhealthy (Group I), gingivitis (Group II) or chronic periodontitis (Group III) with 15 subjects in each category, belonging to both genders. All the subjects were selected from the out-patient division of the department of Periodontics, K.L.E Society's Institute Of Dental Sciences, Bengaluru, Karnataka, India. A written informed consent was obtained from those who agreed to participate voluntarily in the study.

All the subjects enrolled in the study were of both genders, in the age group of 20-50 years, with the presence of at least 20 teeth into the mouth. The participants were classified into three groups depending on periodontal health status (Pradeep AR et al 2009, p.1455): Group I- Absence of clinical signs of gingival inflammation $(\mathrm{GI}=0)$, probing depth $\leq 3 \mathrm{~mm}$, absence of loss of clinical attachment. Group II- Presence of clinical signs of gingival inflammation (GI $>1$ ), probing depth $\leq 3 \mathrm{~mm}$, absence of loss of clinical attachment. Group III- Presence of at least four teeth in each jaw with probing pocket depth of $\geq 5 \mathrm{~mm}$ along with clinical signs of gingival inflammation, clinical attachment loss of $\geq 3 \mathrm{~mm}$, radiographic evidence of alveolar bone loss (No delineation was done within chronic periodontitis based on the extent of alveolar bone loss). Subjects excluded from the study included 1) those with systemic diseases like diabetes, hypertension, heart disease and rheumatoid arthritis that could alter the course of periodontal disease 2) who have taken antibiotic/ anti-inflammatory/ immunosuppressive medication in the previous 6 months of periodontal therapy 3) who have undergone periodontal therapy in the previous 6 months of the study 4) Pregnant women and lactating mothers 5) Smokers and subjects using smokeless tobacco.

Ethical clearance for periodontal intervention \& collection of GCF for the study was obtained from the Institutional Ethics Committee. The assessment of the samples for the detection of sTREM-1 was carried out in the Department Of Microbiology, Kanva Diagnostic Centre, Bengaluru. This study was carried out from October 2014 to March 2015.

\subsection{Clinical examination}

Plaque index (Loe H 1964, p.612), gingival index (Loe H 1963, p.610), Probing pocket depth (PPD) and Clinical attachment level (CAL) were recorded at baseline for all the three groups and at follow-up visits scheduled at 8 weeks following SRP in group II and group III. PPD and CAL were recorded using a constant pressure probe (BrockprobeTM). Orthopantomogram (OPG) for every patient showing clinical signs of attachment loss was taken at baseline. Radiographic bone loss was recorded dichotomously to differentiate subjects with chronic periodontitis from other groups.

\subsection{Collection of GCF}

GCF was collected from all the three groups at baseline and 8 weeks following SRP in groups II and III. Sites selected were: Group I- Multiple sites (three to five sites/subject) to ensure collection of an adequate amount of GCF; Group II- The site with the greatest clinical signs of gingival inflammation in the absence of clinical attachment loss; Group III- The site with the greatest clinical signs of gingival inflammation, greatest probing depth and clinical attachment loss along with radiographic evidence of alveolar bone loss (Pradeep A R et al, 2009, p.1455). GCF was collected by placing a microcapillary pipette at the entrance of the gingival sulcus, gently touching the marginal gingiva. A standard volume of $1 \mu \mathrm{l}$ was collected with an extracrevicular approach (unstimulated) from each test site. Further, micropipettes that were suspected of being contaminated with blood or saliva were discarded. The collected GCF samples were transferred to airtight plastic vials containing $0.5 \mathrm{ml}$ of phosphate buffered saline (PBS) and stored at $-70^{\circ} \mathrm{C}$ until assayed.

\subsection{Analysis of sTREM-1 levels in GCF}

sTREM-1 levels in GCF was determined by ELISA using specific ELISA kit (Raybiotech , Inc USA) which is based on standard sandwich enzyme-linked immunosorbent assay technology. Briefly, samples were incubated with a monoclonal antibody specific for TREM-1 pre-coated onto the wells of a micro plate. Following a wash, to eliminate the unbound substances, an enzyme-linked polyclonal antibody specific for TREM-1 was added to the wells. After washing away the unbound conjugate, a substrate solution was added to the wells. Color development was stopped, and optical density of each well was determined within 30 minutes using a micro plate reader set to $450 \mathrm{~nm}$, with a wavelength correction set to $540 \mathrm{~nm}$. All measurements were performed in duplicate and the sTREM-1 concentration was expressed in picogram/mililiter $(\mathrm{pg} / \mathrm{ml})$.

The mean absorbance for duplicate standards, controls and samples was calculated, and the average zero standard optical density was subtracted from it. A standard curve was plotted, using sigma plot software, with standard concentration on the $\mathrm{x}$-axis and absorbance on the y-axis. (Fig. 1).

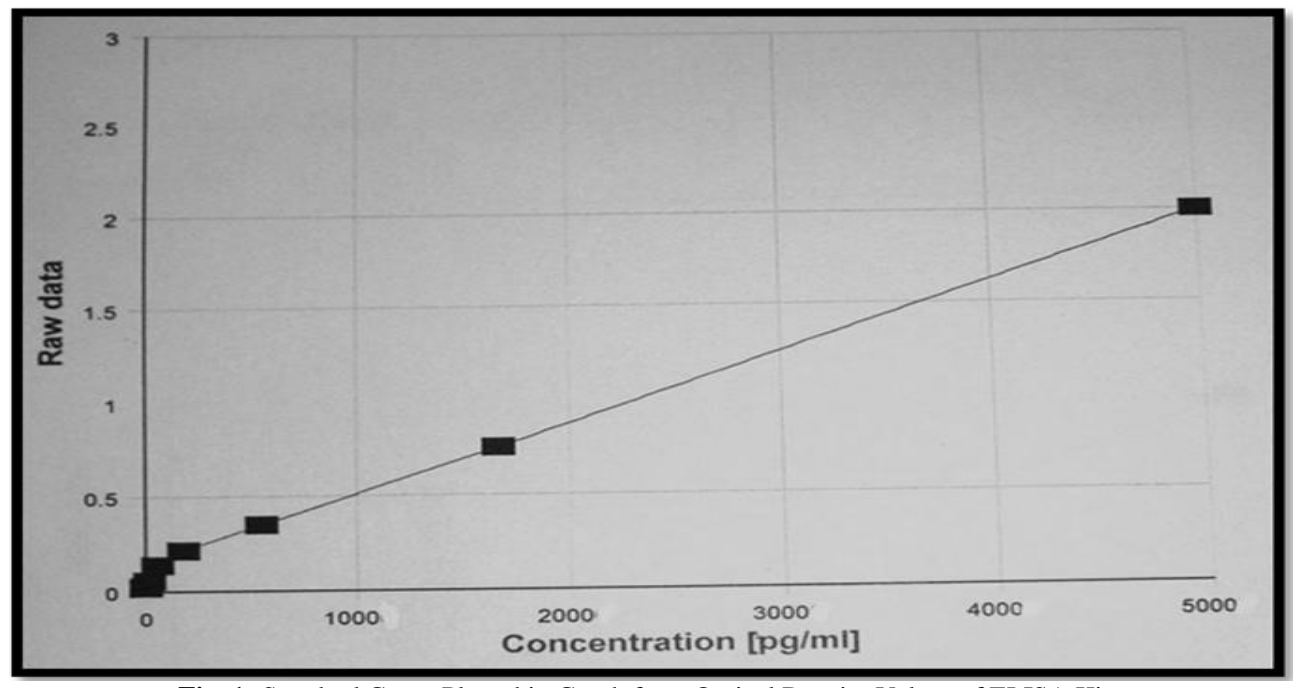

Fig. 1: Standard Curve Plotted in Graph from Optical Density Values of ELISA Kit. 


\subsection{Statistical analysis}

Using PASS (Process Automation Software System) software, sample size was estimated with $90 \%$ power and $5 \%$ level of significance $(\mathrm{p}<0.05)$. Data was entered in Microsoft excel and analysed using SPSS (Statistical Package for Social Science, Ver.10.0.5) package. The results were averaged (mean + standard deviation) for continuous data; number and percentage for dichotomous data were presented in tables and figures.

Chi-square testwas used to determine whether there was a statistical difference between groups in the parameters measured. Pearson correlation coefficients were calculated to determine whether there was any correlation between the STREM-1 level data and the clinical parameters.
$40.47 \pm 7.6$ years and this difference between groups was statistically significant $(\mathrm{p}<0.05)$. However, age is not found to influence sTREM-1 levels in GCF ( $p>0.05)$ [Table 3].

Detectable levels of sTREM-1 were found in GCF samples of all the groups. At baseline, the mean sTREM-1 levels in group I, group II and group III were $69.5 \pm 1.8 \mathrm{pg} / \mathrm{ml}, 257.17 \pm 79.1 \mathrm{pg} / \mathrm{ml}$ and $3658.94 \mathrm{pg} / \mathrm{ml} \pm 13103.5$ respectively; this difference between the groups were statistically significant $(\mathrm{p}<0.05)$ (Fig 2).A reduction was seen from baseline to 8 weeks in group II and group III, with mean sTREM-1 level in group II and group III of 116.38 \pm 38.39 $\mathrm{pg} / \mathrm{ml}$ and $218.18 \pm 113.33 \mathrm{pg} / \mathrm{ml}$ respectively (Fig 3).

Astatistically significant positive correlation was observed between GCF levels of sTREM-1 and GI, PI, PPD and CAL (Fig 4).

\section{Results}

Patient characteristics are summarised in Tables 1 and 2 the study used a wide range of age, where the mean age of group I was 24. $67 \pm 2.5$ years, group II was $33.53 \pm 5.9$ years and group III was

Table 1: Gender Distribution among Study Groups

\begin{tabular}{|c|c|c|c|c|c|}
\hline & $\begin{array}{l}\text { Gender } \\
\text { Male }\end{array}$ & Female & Total & $\chi^{2}$ value & 'p' value \\
\hline Group I & $\begin{array}{l}6 \\
40.0 \%\end{array}$ & $\begin{array}{l}9 \\
60.0 \%\end{array}$ & $\begin{array}{l}15 \\
100.0 \%\end{array}$ & & \\
\hline Group II & $\begin{array}{l}5 \\
33.3 \%\end{array}$ & $\begin{array}{l}10 \\
66.7 \%\end{array}$ & $\begin{array}{l}15 \\
100.0 \%\end{array}$ & & 0.529 \\
\hline Group III & $\begin{array}{l}8 \\
53.3 \%\end{array}$ & $\begin{array}{l}7 \\
46.7 \%\end{array}$ & $\begin{array}{l}15 \\
100.0 \%\end{array}$ & & \\
\hline
\end{tabular}

Table 2: Mean Age Distribution among Study Groups

\begin{tabular}{|c|c|c|c|c|c|c|c|}
\hline & $\mathrm{N}$ & Mean & SD & Min. & Max. & 'F' value & 'p' value \\
\hline Group I & 15 & 24.67 & 2.582 & 20 & 29 & \multirow{3}{*}{27.811} & \multirow{3}{*}{$<0.001$} \\
\hline Group II & 15 & 33.53 & 5.998 & 22 & 42 & & \\
\hline Group III & 15 & 40.47 & 7.671 & 28 & 54 & & \\
\hline
\end{tabular}

Table 3: Comparison of GCF Strem-1 Levels by Age in Study Groups at Baseline and 8 Weeks

\begin{tabular}{|c|c|c|c|c|c|c|c|c|}
\hline Group & Visit & Age & $\mathrm{N}$ & Mean & SD & Min & Max & 'p' value \\
\hline \multirow[t]{5}{*}{ Group I } & \multirow[t]{2}{*}{ Baseline } & $20-29$ yrs & 15 & 69.50 & 1.89 & 68.59 & 73.49 & \multirow[t]{2}{*}{-} \\
\hline & & $20-29$ yrs & 4 & 333.67 & 62.78 & 268.00 & 387.63 & \\
\hline & \multirow{3}{*}{ Baseline } & $30-39$ yrs & 8 & 219.37 & 72.77 & 122.49 & 346.48 & \multirow{3}{*}{0.168} \\
\hline & & $40-49$ yrs & 3 & 255.98 & 43.87 & 205.80 & 287.14 & \\
\hline & & $20-29$ yrs & 4 & 137.07 & 39.93 & 107.30 & 196.00 & \\
\hline \multirow[t]{7}{*}{ Group II } & \multirow[t]{4}{*}{8 weeks } & $30-39$ yrs & 8 & 97.68 & 25.73 & 73.00 & 147.00 & \multirow{4}{*}{0.127} \\
\hline & & $40-49$ yrs & 3 & 138.67 & 51.07 & 97.99 & 196.00 & \\
\hline & & $20-29$ yrs & 1 & 500.00 & & 500.00 & 500.00 & \\
\hline & & $30-39$ yrs & 7 & 1518.20 & 1842.94 & 531.17 & 5555.00 & \\
\hline & \multirow[t]{3}{*}{ Baseline } & $40-49$ yrs & 5 & 5210.72 & 1342.45 & 809.72 & 1667.00 & \multirow[t]{3}{*}{0.295} \\
\hline & & $\geq 50 \mathrm{yrs}$ & 2 & 2996.63 & 3622.03 & 435.47 & 5557.80 & \\
\hline & & $20-29$ yrs & 1 & 435.47 & & 435.47 & 435.47 & \\
\hline \multirow{3}{*}{ Group III } & & $30-39$ yrs & 7 & 180.55 & 81.28 & 97.99 & 339.78 & \multirow{3}{*}{0.106} \\
\hline & 8 weeks & $40-49$ yrs & 5 & 255.89 & 123.49 & 122.49 & 387.63 & \\
\hline & & $\geq 50$ yrs & 2 & 146.99 & 69.30 & 97.99 & 196.00 & \\
\hline
\end{tabular}




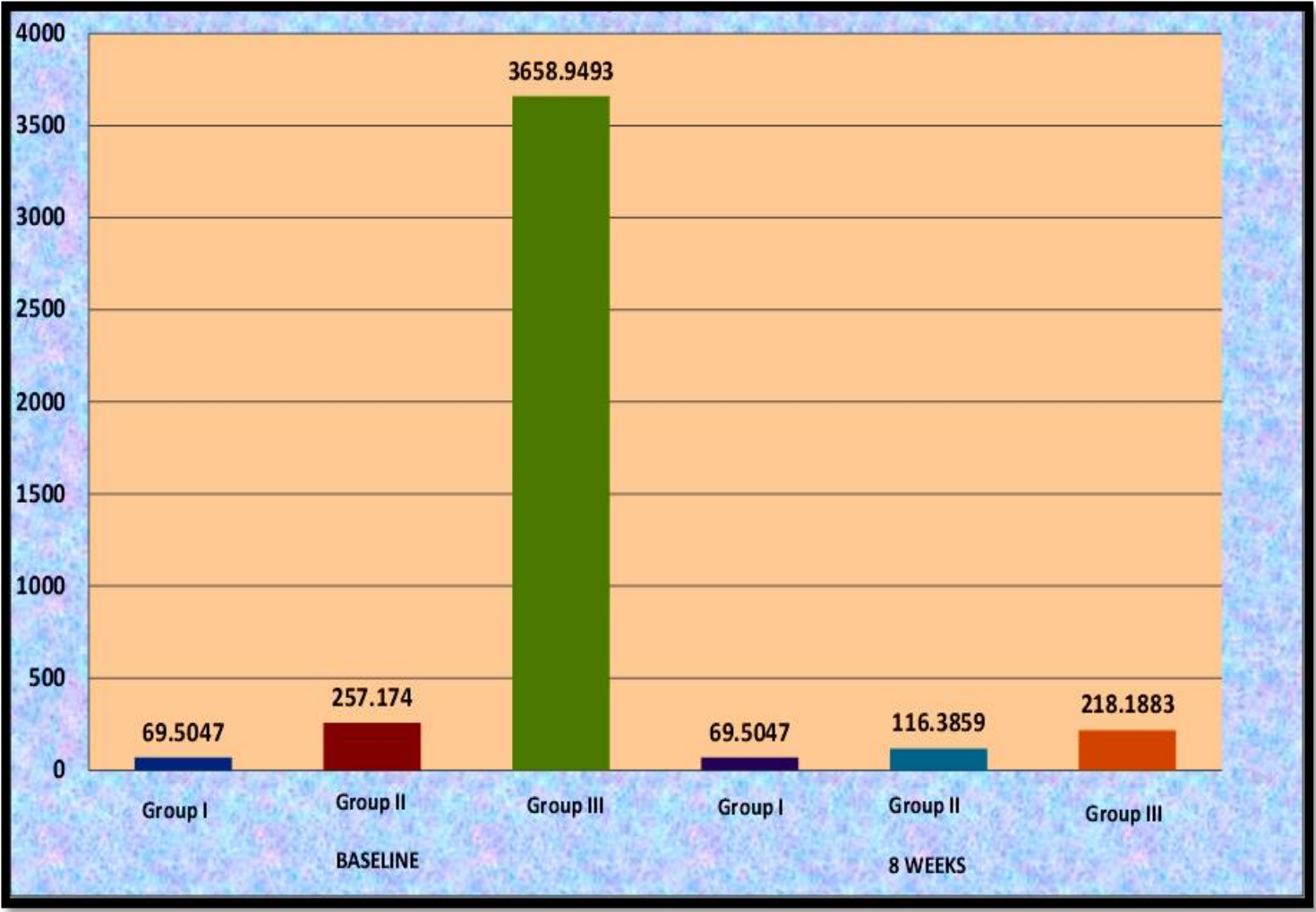

Fig. 2: Comparison of GCF Strem-1 Levels among Study Groups at Baseline and 8 Weeks.

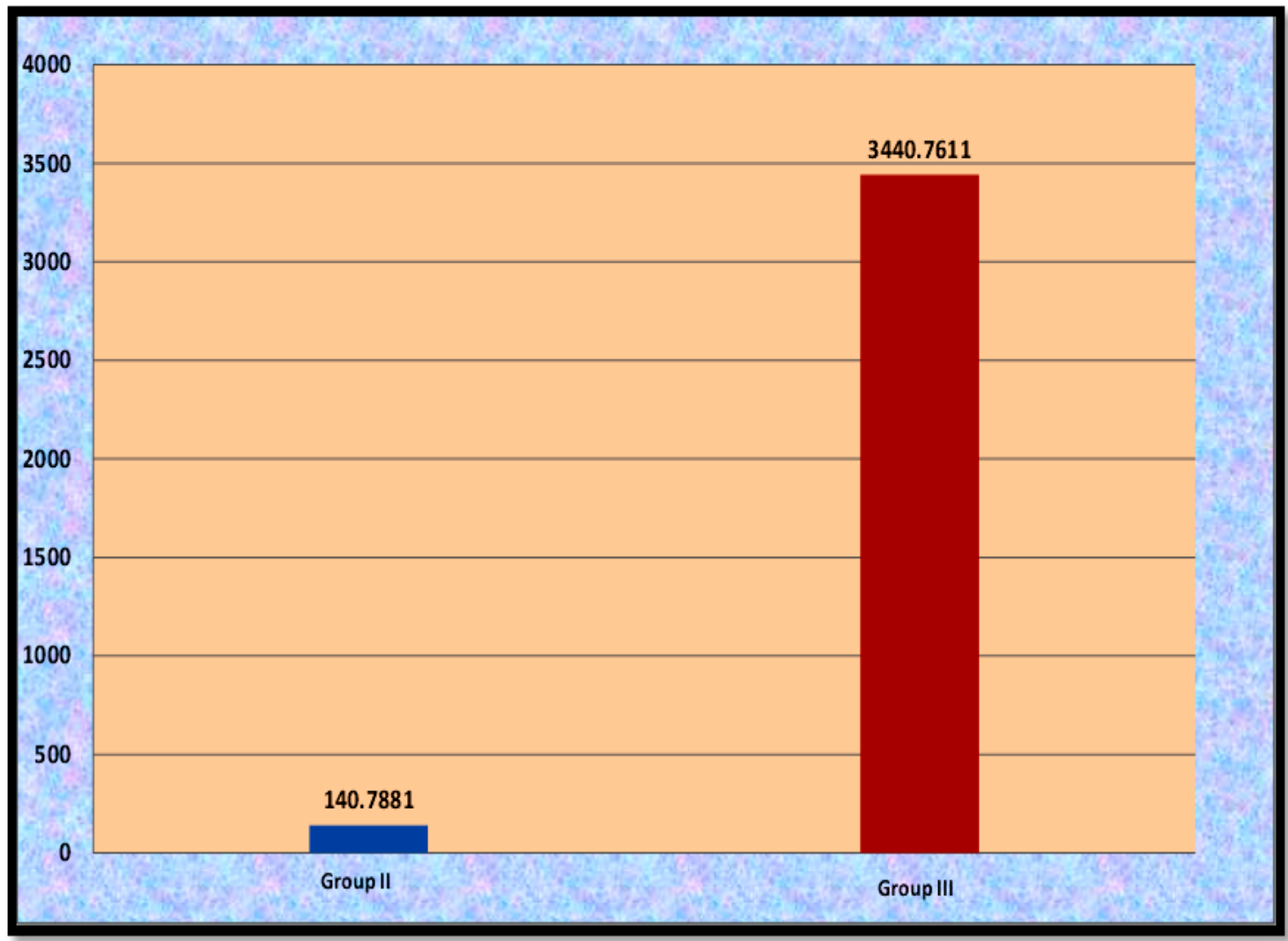

Fig. 3: Comparison of Mean Change of GCF Strem-1 Levels from Baseline among Study Groups. 


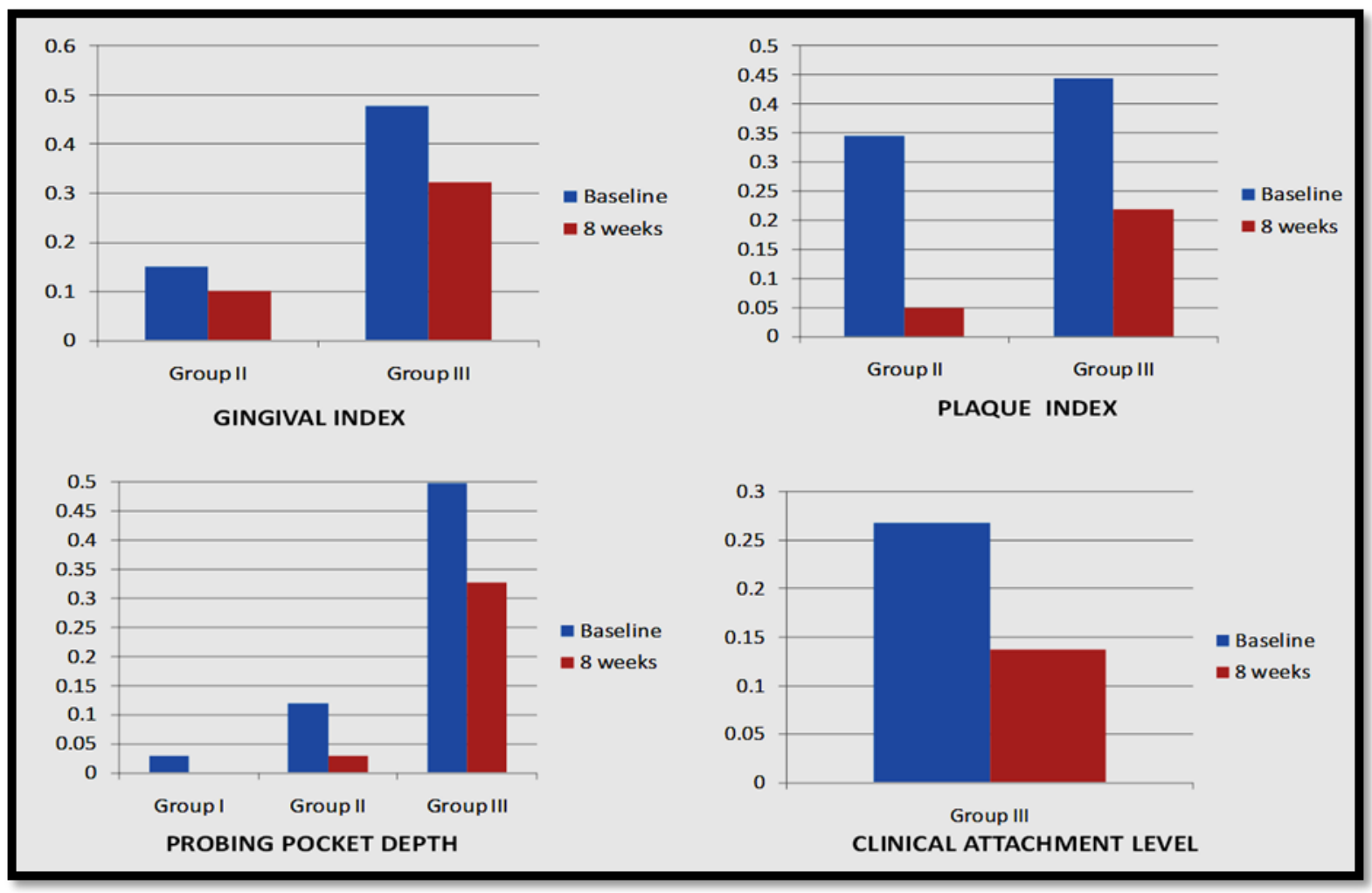

Fig. 4: Correlation of Strem-1 Levels with Clinical Parameters in Study Groups at Baseline and 8 Weeks.

\section{Discussion}

Periodontal diseases are believed to involve intricate interaction of polymicrobial biofilm attaching on tooth surface with the host immune-inflammatory response (Page RC et al 1997, p.216). Innate immune responses against pathogens provide a first line of host defense that occurs rapidly and involves the coordinate action of several cells types such as professional phagocytes and natural killer cells. These cells display a variety of cell-surface receptors that recognize either pathogens or endogenous molecules that are expressed during tissue damage. This innate response is, thus, initiated upon stimulation of pattern recognition receptors such as Toll-like receptors (TLRs) by pathogen-associated molecular patterns (PAMP) and leads to the release of cytokines (TNF- $\alpha$, IL- $1 \alpha$ and IL-6). Recently, another class of cell-surface receptors has been identified:Triggering Receptor Expressed On Myeloid cells (TREM) (Bison C et al 2012, p.1142).

TREM is a cell-surface receptor of the immunoglobulin superfamily which is constitutively expressed by monocytes and polymorphonuclear neutrophils (PMNs). It is activated upon bacterial recognition by host cells, triggering a number of intracellular signalling events that result in enhanced pro-inflammatory cytokine production. These TREM-1 receptors are important regulators of the immune response due to their ability to either amplify or decrease pathogen receptor recognition (PRR)-induced signals"ItInteracts withDNAX activating protein of $12 \mathrm{kDa}$ (DAP12) promotes recruitment and activation of protein tyrosine kinases, leading to tyrosine phosphorylation of many protein species, $\mathrm{Ca} 2+$ mobilization, activation of extracellular signal-regulated kinases (ERK) and transcription complexes downstream of ERK.(Colonna M et al 2003, p.398)

An additional important feature of TREM proteins is their release as soluble forms (sTREM). Soluble TREM may, in fact, be generated by the alternative splicing of mRNA producing secreted receptor isoforms. Otherwise, sTREM may be produced by the cleavage of the extracellular domain off the cell surface (Ford JW et al 2009, p.40).

sTREM-1 amplifies the response induced by toll like receptors, resulting in enhance pro-inflammatory cytokine production. The role of TREM-1 in innate immunity is,thus, the propagation of inflammation in response to microbial infections.

In the study, significantly higher GCF levels of sTREM-1( $p<0.05)$ were observed in periodontitis patients as compared to gingivitis and healthy individuals. sTREM-1 levels were higher in gingivitis patients by 3.7 fold compared to periodontally healthy individuals and higher in periodontitis patients by 5.1 fold compared to gingivitis patients. These findings are well in line with a study by Bison et al, in which GCF sTREM-1 levels were 7 fold higher in pathological sites(periodontitis affected) compared to healthy sites of periodontitis patients, and 14 fold higher in pathological sites(periodontitis affected) compared to non-periodontitis individuals( Bison Cet al 2012, p.1141). Similar results were also observed during the study by Belibasakis et al, where in periodontitis affected sites exhibited significantly higher sTREM-1 levels in GCF than healthy sites, irrespective of the form of periodontitis. This difference was 3.7 fold in the case of chronic periodontitis and 4.5 fold in the case of generalized aggressive periodontitis (Belibasakis GN et al 2014, p. 204).Similarly in a study by Bostanci et al, salivary sTREM-1 concentrations were higher in chronic or aggressive periodontitis than in healthy controls group by 3.3 fold and 5.6 fold respectively. In serum, these differences were 1.7 fold and 2 fold respectively(Bostanci $\mathrm{N}$ et al 2013, p.1).

In the present study, a positive correlation was observed between GCF sTREM-1 levels and all clinical parameters including PI, GI, PPD and CAL in the study groups. This finding supports the role of sTREM-1 in the propagation of inflammation in response to microbial infections. Further evidence to support this comes from the positive correlation of sTREM-1 levels with bleeding on probing (BOP), a finding backed up by the earlier demonstration of a positive correlation with gingival index scores (Belibasakis GN et al 2014, p.208).

A wide variation was found in STREM-1 concentrations among patients in group II and group III. In group II, highest sTREM-1 level was $387.15 \mathrm{pg} / \mathrm{ml}$ and lowest was $167 \mathrm{pg} / \mathrm{ml}$; in group III, the highest sTREM-1 level was $5557.8 \mathrm{pg} / \mathrm{ml}$ and lowest was $435.47 \mathrm{pg} / \mathrm{ml}$. This variability of sTREM-1 concentration among the patients can be attributed to either the differences in disease stage at the time of GCF sampling or the episodic nature of the periodontal destruction. 
The most common form of initial treatment for periodontal diseases is scaling and root planing along with oral hygiene instructions. There is considerable evidence supporting SRP as an essential and effective component of periodontal therapy (Hammerle CHF et al, 1991; Cobb CM, 1996; Hill RW et al, 1981; Kalashl WB et al, 1988). In contrast to previous studies(Bison C et al, 2011; Bostanci N, 2012; Bostanci N et al, 2013;Belibasakis GN et al, 2014 Bostanci $\mathrm{N}$ et al, 2013), the present study has evaluated the effect of non-surgical periodontal therapy (SRP) on GCF sTREM-1 levels, which might have further confirmed the role of sTREM-1 in periodontal disease. In this study, the levels of sTREM-1 were found to decrease significantly $(\mathrm{p}<0.05)$ after non-surgical periodontal therapy (SRP) in groups II(gingivitis) and III (chronic periodontitis). The mean reduction from baseline in group II was $140.78 \mathrm{pg} / \mathrm{ml}$ and $3440.76 \mathrm{pg} / \mathrm{ml}$ in group III. Furthermore, there was a significant overlap in sTREM-1 levels between group II and group III post SRP, possibly due to the resolution of periodontitis after treatment. In the present study, SRP led to the significant improvement in all clinical parameters including PI, GI, PPD and CAL.

In the present study, Brockprobe ${ }^{\mathrm{TM}}$ was used to record probing pocket depth and clinical attachment level.Brockprobe ${ }^{\mathrm{TM}}$ is a second generation pressure sensitive probe with William's markings $(1,2,3,5,7,8,9,10 \mathrm{~mm}$ ) (Veena HR et al 2010, p.41). The probe exerts a standardized pressure of 20 grams. This consistent pressure assures that the instrument will work in the same manner and give the same results, time after time.

In the present study, periodontitis patients were not subclassified based on probing depth, which would have added more value in confirming the association between sTREM-1 and periodontal inflammation.

The present study has estimated the levels of GCF sTREM-1 in healthy, gingivitis and periodontitis patients at baseline and 8 weeks following SRP in gingivitis and periodontitis patients. Subgingival microbial repopulation occurs within a few months (2 months) after instrumentation of periodontal pockets. Since, sTREM-1 is rapidly activated upon bacterial challenge, microbial analysis would have added more value to the results.

The relatively high GCF levels of sTREM-1 in periodontitis patients confirmed the role of TREM- 1 in periodontal tissue destruction. It may be considered as an inflammatory biomarker of periodontal disease and can serve both as a diagnostic biomarker and predictor of future disease initiation and progression. Further research is needed to determine the role of sTREM-1 in the evolution from the initial immunological events occurring in the inflamed periodontal tissue to the development of inflammatory periodontal disease with bone destruction.

\section{Conflict of interest}

The authors have declared that no competing interests exist.

\section{References}

[1] Anitha K, Balu P, Sakthidevi S, Kumar A. Biomarkers-A diagnostic tool for periodontal diseases. Journal of Scientific Dentistry 2013; 3: 59-65.

[2] Bostanci N, Thurnheer T, Belibasakis GN. Involvement of the TREM-1/DAP12 pathway in the innate immune response to Porphyromonasgingivalis. MolImmunol 2011; 49:387-394. https://doi.org/10.1016/j.molimm.2011.09.012.

[3] Bison C, Massin F, Lefevre PA, Thelly N, Miller N, Gibot S. Increased gingival crevicular fluid levels of soluble triggering receptor expressed on myeloid cells sTREM-1 in severe periodontitis. J ClinPeriodontol 2012; 39:1141-1148. https://doi.org/10.1111/jcpe.12008

[4] Bostanci N, Belibasakis GN. Doxycycline inhibits TREM-1 induction by Porphyromonasgingivalis. FEMS Immunol Med Microbiol2012; 66:37-44. https://doi.org/10.1111/j.1574695X.2012.00982.x.

[5] Bostanci N, Thurnheer T, Opoku J, Curtis MA, Zinkernagel AS, Belibasakis GN. Porphyromonasgingivalis regulates TREM-1 in human polymorphonuclear neutrophils via its gingipains. Plos One 2013; 8:1-8. https://doi.org/10.1371/journal.pone.0075784.

[6] Lei L, Li H, Yan Fuhua, Xiao Y. Hyperlipidemia impaired innate immune response to periodontal pathogen Porphyromonasgingivalis in apolipoprotein E knockout mice. Plos One 2013; 88:1-9.

[7] Willi M, Belibasakis GN, Bostanci N. Expression and regulation of TREM-1 in periodontal diseases. ClinExpImmunol 2014; 178:190200. https://doi.org/10.1111/cei.12397.

[8] Pradeep AR, Happy D, Parag H, Garima G, Manojkumar T. Correlation of gingival crevicular fluid interleukin-18 and monocyte chemoattractant protein-1 levels in periodontal health and disease. $\mathrm{J}$ Periodontol 2009 https://doi.org/10.1902/jop.2009.090117.

[9] Loe H. The gingival index, the plaque index and the retention index $\begin{array}{llll}\text { systems. } & \mathrm{J} & \text { 38:610-616 }\end{array}$ https://doi.org/10.1902/jop.1967.38.6 part2.610.

[10] Colonna M, Facchetti F. TREM-1(Treiggering Receptor Expressed on Myeloid Cells): a new player in acute inflammatory responses. JID 2003; 187(2):397-401. https://doi.org/10.1086/374754.

[11] Ford JW, McVicar DW. TREM and TREM-like receptors in inflammation and disease. CurrOpinImmunom 2009; 21 (1): 38-46. https://doi.org/10.1016/j.coi.2009.01.009.

[12] BelibasakisGN,Ozturk VO, EmingilG,Bostanci N. Soluble triggering receptor expressed on myeloid cells (sTREM-1) in gingival crevicular fluid: Association with clinical and microbiological parameters. J periodontol 2014; 85:204-210. https://doi.org/10.1902/jop.2013.130144.

[13] Bostanci N, Ozturk VO, Emingil G, Belibasakis GN. Elevated oral and systemic levels of soluble triggering receptor expressed on myeloid cells -1 in periodontitis. J Dent Res 2013; 92:161-165 https://doi.org/10.1177/0022034512470691.

[14] Hammerle CHF, Joss A, Lang NP. Short term effects of initial periodontal therapy (hygienic phase). J ClinPeriodontol1991; 18: 233239 https://doi.org/10.1111/j.1600-051X.1991.tb00420.x

[15] Cobb CM. Non-surgical pocket therapy: mechanical. Ann Periodontol 1996; 1:443-490. https://doi.org/10.1902/annals.1996.1.1.443.

[16] Hill RW, Ramfjord SP, Morrison EC. Four types of periodontal treatment compared over two years. J Periodontol 1981; 52:655662. https://doi.org/10.1902/jop.1981.52.11.655.

[17] Kaldahl WB, Kalkwarf KL, Patil KD, Dyer JK, Bates RE Jr. Evaluation of four modalities of periodontal therapy. Mean probing depth, probing attachment level and recession changes. J Periodontol 1988 59:783-793. https://doi.org/10.1902/jop.1988.59.12.783.

[18] Veena HR, Prasad D. Evaluation of an aminobisphosphonate (alendronate) in the management of periodontal osseous defects. J Indian SocPeriodontol. 2010; 14: 40-45. https://doi.org/10.4103/0972124X.65438 